\title{
CAPÍTULO 02: PRINCIPAIS CAUSAS DA GRAVIDEZ NA ADOLESCÊNCIA UM PROBLEMA DE SAÚDE PÚBLICA: REVISÃO INTEGRATIVA DE LITERATURA
}

\author{
CAPÍTULO 02: PRINCIPALES CAUSAS DEL EMBARAZO EN LA \\ ADOLESCENCIA UN PROBLEMA DE SALUD PÚBLICA: REVISIÓN \\ INTEGRATIVA DE LA LITERATURA
}

\section{CHAPTER 02: MAIN CAUSES OF PREGNANCY IN ADOLESCENCE A PUBLIC HEALTH PROBLEM: INTEGRATIVE LITERATURE REVIEW}

Nycolle Santana dos Santos ${ }^{1}$; Levi Araujo Bezerra ${ }^{2}$; Kaline Rafaelle Dias da Silva ${ }^{3}$; Luiz Carlos Alves da Silva ${ }^{4}$;

DOI: https://doi.org/10.31692/978-65-88970-04-1.15-30

\begin{abstract}
RESUMO
$\mathrm{O}$ índice de adolescentes que engravidam no Brasil, 65,5 em cada mil, estando acima da média mundial que é 46 a cada mil. Levando em consideração que uma gravidez na adolescência pode causar morte e invalidez, é necessário pontuar que é merecida uma atenção diferenciada por caracterizar-se como um problema de saúde pública. Este estudo teve por objetivo analisar as principais causas da gravidez na adolescência e a existência de políticas públicas específicas, foi realizada uma revisão integrativa de literatura onde consta nas bases de dados LiLACS, MEDLINE, BDENF, SCIELO e BVS, no período de 2010-2019, sendo usados os seguintes descritores: gravidez na adolescência, políticas públicas e adolescente. Os critérios de inclusão foram: artigos de pesquisa original; completos e disponíveis para leitura/análise; escritos em língua portuguesa. Foram selecionados 14 artigos com aderência ao tema. A gravidez na adolescência está frequentemente relacionada a questões sociais e econômicas, sendo o casamento uma opção desejável para a família, muitas vezes em condições economicamente desfavoráveis, o mesmo pode ser a saída pela falta de estudos e oportunidades de emprego, sendo uma alternativa para assegura o futuro financeiro. Estudos também apontaram que quanto mais cedo a jovem inicia a vida sexual, maiores são as probabilidades de engravidar precocemente, a despeito do conhecimento já produzido sobre políticas públicas específicas de Juventude, não há políticas para esse grupo, foi identificada a Estratégia de Saúde da Família no contexto de saúde das jovens. Porém, é necessária a incorporação de informações sobre o atendimento às individualidades que envolvem o adolescente e a disponibilidade de informações sobre as necessidades dos adolescentes e a prevenção da gravidez.
\end{abstract}

Palavras-Chave: Gravidez; políticas públicas; adolescência.

\section{RESUMEN}

La tasa de adolescentes que quedan embarazadas en Brasil, 65,5 por mil, está por encima del promedio mundial, que es de 46 por mil. Teniendo en cuenta que un embarazo en la adolescencia puede ocasionar la muerte y la discapacidad, es necesario señalar que se merece una atención diferenciada por ser caracterizado como un problema de salud pública. Este estudio tuvo como objetivo analizar principales causas del embarazo en la adolescencia y la existencia de políticas públicas específicas, realizó una revisión integradora de la literatura que aparece en las bases de datos LiLACS, MEDL/ BDENF, SCIELO y BVS, en el período 2010-2019, utilizando los siguientes descriptores: emb adolescente, políticas públicas y adolescente. Los criterios de inclusión fueron: artícu investigación originales; completo y disponible para lectura / análisis; escrito en portu

\footnotetext{
${ }^{1}$ Enfermagem, UFPE - CAV (Universidade Federal de Pernambuco - Centro Acadêmico de Vitória) nycolle005@gmail.com

${ }^{2}$ Ciências Biológicas, UFPE - CAV (Universidade Federal de Pernambuco - Centro Ac levitj1.lab@gmail.com

${ }^{3}$ Direito, UNICAP - (Universidade Católica de Pernambuco), kalinedias19@gmai

${ }^{4}$ Mestre em educação agrícola, IFPE (Instituto Federal de Educação, ciência nagusto.eaf@hotmail.com 
seleccionaron 14 artículos con apego al tema. El embarazo adolescente suele estar relacionado con cuestiones sociales y económicas, siendo el matrimonio una opción deseable para la familia, muchas veces en condiciones económicamente desfavorables, lo mismo puede ser la salida por la falta de estudios y oportunidades laborales, siendo una alternativa para asegurar el futuro financiero. Estudios también señalaron que cuanto antes la joven inicia la vida sexual, mayores son las posibilidades de quedar embarazada precozmente, a pesar del conocimiento ya producido sobre políticas públicas específicas para la Juventud, no existen políticas para este colectivo, se identificó la Estrategia Salud de la Familia. en el contexto de la salud de las mujeres jóvenes. Sin embargo, es necesario incorporar información sobre la atención brindada a las personas que involucran a adolescentes y la disponibilidad de información sobre las necesidades de las adolescentes y la prevención del embarazo.

Palabras Clave: El embarazo; políticas públicas; adolescencia.

\section{ABSTRACT}

The rate of adolescents who become pregnant in Brazil, 65.5 per thousand, being above the world average, which is 46 per thousand. Taking into account that a teenage pregnancy can cause death and disability, it is necessary to point out that differentiated attention is deserved for being characterized as a public health problem. This study aimed to analyze the main causes of pregnancy in adolescence and the existence of specific public policies, an integrative literature review was carried out in the LiLACS, MEDLINE, BDENF, SCIELO and VHL databases, in the period of 2010-2019, using the following descriptors: teenage pregnancy, public policy and adolescent. Inclusion criteria were: original research articles; complete and available for reading / analysis; written in Portuguese. 14 articles were selected with adherence to the theme. Teenage pregnancy is often related to social and economic issues, with marriage being a desirable option for the family, often in economically unfavorable conditions, the same can be the way out due to the lack of studies and job opportunities, being an alternative to ensure the financial future. Studies also pointed out that the sooner the young woman starts sexual life, the greater the chances of becoming pregnant early, despite the knowledge already produced about specific public policies for Youth, there are no policies for this group, the Family Health Strategy was identified in the context of young women's health. However, it is necessary to incorporate information about the care provided to individuals that involve adolescents and the availability of information about adolescents' needs and pregnancy prevention.

Keywords: Pregnancy; public policy; adolescence.

\section{INTRODUÇÃO}

A palavra adolescência originou-se do latim que, etimologicamente, significa ad (para, a) e olescer (crescimento), também deriva de adoecer, esses significados retratam o crescimento como um processo duradouro, doloroso, fisicamente e psicologicamente (VIOLA; VORCARO, 2018). A adolescência é definida como o período de tempo entre a infância e a fase adulta, um estágio de transição que traz consigo mudanças significativas, onde vai interferir diretamente em aspectos emocionais, comportamentais e biológicos (MARTINEZ et al.,2011; MATTOS, 2011).

Apesar da delimitação pouco precisa, Arnett (2000), aponta que este período se inicia com a puberdade e finaliza com a entrada do jovem na vida adulta, quando ele, então, assume responsabilidades e deveres estabelecidos por sua cultura.

As rápidas mudanças que ocorrem no desenvolvimento humano durante a adolescência exigem adaptações constantes do indivíduo e dos contextos nos quais ele se insere, sendo uma 
preparação para a fase madura da vida, em que o início desta não tem um ponto especifico, mas sabe-se que está acabando quando se está como no final de uma luta penosa (BERNI; ROSO, 2014).

Erikson (1976) em seu livro Identidade, juventude e crise, corrobora com Berni e Roso, (2014), ao associar o período da adolescência a constantes lutas psicológicas inerentes à formação da personalidade. Ele escreve referindo-se à adolescência como:

\footnotetext{
"É um período da vida em que o corpo muda radicalmente de proporções, a puberdade genital muda o corpo e a imaginação com toda espécie de impulsos, a intimidade com o outro sexo se inicia e o futuro imediato o coloca diante de um número excessivo de possibilidades e escolhas conflitantes [...] ele deve fazer uma série de seleções cada vez mais específicas de compromissos pessoais, ocupacionais, sexuais e ideológicos" (ERIKSON, 1968, p. 132-245).
}

Por esse ser um período de intensas contradições e confronto de ideias de oposição aos valores, tradições e regras sociais, os adolescentes podem estar vulneráveis a comportamentos não saudáveis, estando, mais expostos a danos à saúde, pois os adolescentes frequentemente não se submetem as normas sociais, sendo sua existência regida por suas próprias regras, o que favorece o uso de drogas, álcool e a prática de sexo desprotegido (FIEDLER; ARAÚJO; SOUZA, 2015).

Por ser uma fase da vida marcada por episódios de transgressão a regras sociais, é comum que a primeira experiência sexual ocorra na adolescência, sendo comum a ocorrência de gravidez nessa fase. Em 2007, a proporção de nascimentos no Brasil cujas mães tinham idade entre 10 a 19 anos, foi de $21,1 \%$. Sendo o parto entre adolescentes a primeira causa de internação hospitalar do sexo feminino (BRASIL, 2008).

Segundo Dias e Teixeira (2010), até meados do século XX, a gravidez na adolescência não era considerada uma questão de saúde pública, e por esse motivo, não era foco de estudo como atualmente, tornando-se mais visível com o aumento do número de nascimentos em mãe menores de 20 anos no decorrer da década de 90, quando os percentuais passaram de 16,38\% em 1991 para 21,34\% em 2000.

Passar pela fase materna, ao mesmo tempo em que é adolescente, não é uma experiência fácil, as transformações pelas quais as adolescentes passam nesse período do desenvolvimento, faz com que apresentem dificuldades além das que costumam surgir para qualquer grávida, ao desempenhar o papel materno, uma vez que não dispõem da maturidade psicológica necessária para suprir as demandas diárias da maternidade (OLIVEIRA, 2018). 
A maternidade exige que a futura mãe redefina toda sua vida, da gravidez em diante, estando vinculada às demandas do filho, os elementos e experiências importantes da construção de sua identidade na adolescência são afetados, pois toda sua vida será reajustada. Tendo em base todas as complicações envolvidas com a gravidez na adolescência, o presente estudo teve por objetivo analisar as principais causas da gravidez na adolescência e a existência de políticas públicas específicas.

\section{FUNDAMENTAÇÃO TEÓRICA}

Para Brito (2011); Erikson (1968) e MARTINEZ et al. (2011) a adolescência inicia com mudanças, caracterizadas por momentos de profundas transformações, este período marca o início da vida reprodutiva, sendo caracterizado por mudanças corporais que também repercutem no psicológico e no emocional do indivíduo. É nesta fase de extrema relevância que o adolescente irá adquirir subsídios que irão consolida seu crescimento e sua personalidade (FILIPINI et al., 2013).

A Organização Mundial da Saúde (OMS), considera a adolescência como o período compreendido entre os 10 e 19 anos (WHO, 2001). No Brasil, o Estatuto da Criança e do Adolescente (ECA), Lei 8.069, de 1990, considera criança a pessoa até 12 anos de idade incompletos e define a adolescência como a faixa etária de 12 a 18 anos de idade (artigo 2o), e, a depender da situação e em casos excepcionais quando disposto na lei, o estatuto é aplicável até os 21 anos de idade (BRASIL, 1990).

Eisenstein (2005), em seu estudo sobre definições e conceitos da adolescência, afirma que, é importante observar a variabilidade no tempo de início, duração e progressão da adolescência, com marcantes diferenças entre os sexos e entre os diversos grupos étnicos e sociais de uma população, inclusive o estado nutricional e fatores familiares, ambientais e contextuais.

Brêtas (2003), em sua tese de doutorado discorre sobre a diferenciação adolescência e puberdade, não são sinônimas, pois a puberdade é apenas um dos aspectos dessa fase e refere-se ao processo de desenvolvimento orgânico e corporal, ocorrendo entre os 9 e 14 anos de idade para os meninos, e entre os 8 e 13 para as meninas. Essas transfornaçõoes são provocadas por alterações hormonais como o estrógeno e progesterona testosterona nos meninos, podendo durar até os 20 anos de idade.

Pontuando sobre a influência dos hormônios nas transformaçôes do corpo durante a adolescência, Brêtas (2003) escreve que: 


\begin{abstract}
"Uma área do cérebro, chamada hipotálamo, envia uma mensagem para uma glândula chamada hipófise ou pituitária. A hipófise começa a produzir hormônios relacionais ao crescimento (FSH, LH e outros), que são lançados no sangue e cujo efeito estimula outras glândulas endócrinas do corpo, tais como as glândulas sexuais e faz com que elas também produzam hormônios. O hormônio folículo-estimulante (FSH) atua no desenvolvimento dos folículos no ovário e, atuando em combinação com o hormônio luteinizante (LH), regula a ovulação na mulher e a secreção de estrógeno pelo ovário. No homem, atua no desenvolvimento dos testículos e a secreção de andrógeno; influencia especificamente as fases finais da espermatogênese. O hormônio LH atua sobre o ovário, estimulando a produção de progesterona. Combinado com o hormônio folículo-estimulante, regula a ovulação e a secreção ovariana de estrógeno. No homem, estimula a evolução do tecido intersticial (células intersticiais de Leydig), a secreção intersticial e a secreção de andrógeno". (BRÊTAS, 2003).
\end{abstract}

De acordo com Chaui (1987), essas transformações levam o adolescente a querer viver sua sexualidade, muitas vezes sem proteção. Nessa fase, é comum observar a oposição a ideias e valores pela necessidade de contrariar as normas impostas pela sociedade em busca de novas experiências, pois o adolescente acredita ser ilimitado, o que o torna vulnerável tanto física como intelectualmente (FILIPINI et al., 2013).

Filipini et al. (2013), também aponta que é nessa fase onde a sexualidade é estruturada e consolidada, com isso é de grande importância pontuar que as ações no aspecto sexual, realizadas de forma irresponsável provoca conflitos e mudanças na expectativa de futuro do adolescente, podendo causar situações, muitas vezes, irreparáveis como uma gravidez não planejada, aborto, evasão escolar e possíveis problemas de saúde resultantes do sexo desprotegido.

No estudo realizado por Costa e Fernandes (2012), foi analisada a visão de adolescentes sobre amor, sexo e afetividade, ficando evidenciado o descompromisso dos indivíduos de um para o outro, denotando a ideia de prazer por prazer, onde 10,6\% dos adolescentes evocavam sexo como orgia, autossatisfação, medo da solidão ou rejeição, experimentação, ensaio. autoafirmação, curiosidade e um meio de gratificação imediata.

A iniciação precoce da pratica de sexo desprotegido, ao mesmo tempo que permite adentrar em um mundo de novas descobertas, pode levar a diversas complicações de saúde, como infecções sexualmente transmissíveis, também a ocorrência de gravidez na adolescência, aborto e outros problemas de ordem biopsicossocial, como explanado no estudo de Silva, et al, (2015). 
Outro dado importante apresentado por Silva e colaboradores, foi o fato de que 49,25\% dos adolescentes investigados, haviam iniciado sua vida sexual antes dos 15 anos de idade, está informação é semelhante as apresentadas em outros estudos, que revelaram o início das vivencias sexuais nessa idade (ONYEONORO, et al., 2011; SILVA, et al, 2015; VANZIN, et al., 2013).

Segundo Martinez et al. (2011), por muito tempo, a adolescência foi considerada a etapa ideal para engravidar, pois essa também era a idade adequada para casar, mas, hoje é considerada inadequada, perante as associações da gravidez precoce com morbidades do neonato e impactos econômicos, educacionais e sociais. Essa associação tem por mecanismos fatores como a imaturidade do sistema reprodutivo o ganho de peso inadequado durante a gestação, além de aspectos como a pobreza, falta de instrução e cuidados pré-natais.

Nesse contexto, a gravidez na adolescência seria uma experiência indesejada, visto que restringiria as possibilidades de exploração de sua identidade e preparação para o futuro profissional. Em função disso, a gravidez na adolescência passou a ser vista como um fator de risco, capaz de trazer consequências negativas para as adolescentes e o grupo social onde está inserida (DIAS; TEIXEIRA, 2010).

Tornando-se, a gravidez um problema social e de saúde pública, sendo vista como um desvio de percurso, supostamente não desejado pelas adolescentes e cujas consequências frustram o que seria considerado uma adolescência saudável ou "normal" dentro do possível. Silva e Surita (2012) em seu estudo, citam a existência de desvantagens para a saúde de gestantes adolescentes e seus filhos, atribuída à condição biológica de imaturidade, significativamente mais presente em comparação aos resultados encontrados para mulheres adultas, entre 20 e 30 anos, reconhecidamente o período mais apropriado para a maternidade em todas suas dimensões.

Um dado apresentado pelo ministério da saúde, aponta que o número de partos entre 10 a 19 anos, realizados pelo Sistema Único de Saúde, estão reduzindo em mais de 22,0\% segunda metade da década passada, e entre 2000 e 2009, após diminuição de 15,6\% na primeira metade, reduzindo em 34,6\% em todo o período (BRASIL, 2008).

Porém, dados de estudos realizados pelo conselho nacional de pesquisá norteamericano, mostram que houve declínio contínuo e consistente entre 1991 e 2005, com aumento nos entre 2007 e 2009 , correspondendo a 0.5 por 1.000 , entre 10 e 14 anos, e 39, 1 por 1.000 , entre 15 e 19 anos, respectivamente (LLOYD, 2005).

De acordo com Silva e Surita (2012), a gravidez neste período, mostra-se, como um problema não resolvido nos países em desenvolvimento e em alguns dos chamados 
desenvolvidos, o conceito prevalente é de que sua maior causa reside nos aspectos sociais relacionados, e não nos biológicos ou médicos como se acreditava anteriormente, fato aceito de modo equivocado até hoje.

\section{METODOLOGIA}

Trata-se de uma revisão integrativa de literatura a qual se caracteriza em um método de pesquisa utilizado com frequência baseado em evidencias cujo objetivo é reunir e sistematizar resultados anteriores de pesquisas sobre um determinado tema ou questão, de maneira sistemática e ordenada, a fim de apresentar, discutir e aprofundar conhecimentos acerca da temática proposta e as conclusões são estabelecidas mediante a avaliação crítica de diferentes abordagens metodológicas (SOUZA, 2010).

O presente estudo teve como questão norteadora: "Quais as principais causas da gravidez na adolescência, e se existem políticas públicas específicas para essa população?”. Tendo por objetivo, analisar as principais causas da gravidez na adolescência e a existência de políticas públicas específicas.

As etapas que conduziram esta revisão integrativa foram: a elaboração da questão norteadora, definição das bases de dados e estabelecimento de critérios para inclusão e exclusão de estudos/amostragem ou busca na literatura, definição das informações a serem extraídas dos estudos selecionados, avaliação dos estudos incluídos na revisão integrativa, interpretação dos resultados e por fim, apresentação da síntese do conteúdo.

Os artigos foram identificados por busca bibliográfica realizada entre os anos de 2010 a 2020, nas seguintes bases de dados: Medical Literature Analysis and Retrieval System Online (MEDLINE), consultada por meio da Base de Dados Específica da Enfermagem (BDENF), e Literatura Latino-Americana e do Caribe em Ciências da Saúde (LILACS), consultados pela Biblioteca Virtual em Saúde (BVS).

Os artigos selecionados respeitaram os critérios de inclusão e exclusão descritos a seguir, foram analisados os artigos científicos escritos no idioma português, publicados entre os anos de 2010 a 2019, que estivessem publicados na íntegra e adequados aos objetivos. Foram excluídos os artigos escritos em outros idiomas, publicados antes de 2010, que não estivessem disponíveis na integra e não fossem adequados aos objetivos deste estudo.

A estratégia de busca na base MEDLINE utilizou os seguintes descritores (DeCS): gravidez na adolescência, causas da gravidez, adolescência e políticas públicas, estratégias equivalentes foram adotadas para as demais bases. 
Para a discussão, foi realizada uma nova busca a partir do material encontrado nos artigos inseridos nos critérios de inclusão. Outras referências foram utilizadas, como livros e artigos que abordassem o tema gravidez na adolescência, sendo consideradas as informações importantes relativas ao tema em estudo.

\section{Procedimento}

As bibliográficas apresentadas após a buscas com os descritores e critérios de inclusão foram analisadas segundo as seguintes etapas:

1- Leitura exploratória: Uma leitura rápida com o objetivo de analisar se os materiais encontrados estão enquadrados nos critérios do estudo:

2- Leitura seletiva: Leitura das partes mais importantes dos artigos feita de maneira mais aprofundada;

3- Registro dos dados retirados das fontes: Inicialmente foi realizado um agrupamento dos artigos que foram escolhidos, com os nomes dos autores, ano de publicação, objetivo do estudo, resultados e conclusão. Em seguida foram produzidas tabelas com os autores estudados de cada fonte de dado utilizada.

A análise dos dados ocorreu por meio de uma leitura sistemática e criteriosa dos artigos selecionados, para que fossem identificadas as informações necessárias para a produção do presente estudo.

Diante disso foi realizada uma comparação entre os resultados obtidos e uma discussão acerca do que foi encontrado. A análise deste estudo foi descritiva, onde os resultados foram transcritos de forma dissertativa, de acordo com o que foi constatado na leitura.

\section{RESULTADOS E DISCUSSÃO}

Na busca realizada em todas as fontes acima citadas, foram apontados inicialmente 358 estudos, após a leitura exploratória dos resumos de cada um e verificação do enquadramento nos critérios deste estudo, foram selecionados na LILACS 5 artigos, na BDENF 5 artigos. SCIELO 13 e na MEDLINE 207 artigos responderam aos descritores, mas, não atendiam aos critérios de inclusão, porém 4 dos 13 artigos encontrados na SCIELO correspondia aos mesmos resgatados na LILACS e ou na BDFENF, um dos artigos encontrados na SCIELO que atendeu aos critérios de seleção, apresentou indisponibilidade de recuperação na íntegra.

Em seguida, todos os 14 , foram lidos individualmente para confirmação de adequação 
aos critérios de seleção. Um roteiro de coleta foi utilizado para auxiliar no registro das informações identificadas em cada estudo, contendo os seguintes tópicos sobre cada artigo: identificação, objetivo, características metodológicas, principais resultados e conclusões do estudo.

De acordo com a análise realizada nos artigos selecionados, no quadro abaixo, estão relacionados os dados gerais dos estudos, como os nomes dos autores, ano de publicação, objetivo e tipo de estudo.

Quadro 1. Descrição dos artigos selecionados.

OBJETIVO

METODOLOGIA

AUTOR/ANO

Carmo, Livramento, Neto, Zeferino, 2014.

Souza, Burgardt, Ferreira, Bub, Monticell, Haimée Emerich Lentz, 2010.

Monteiro, Freitas, Farias, 2014

Torres; Torres; Vieira;

Barbosa; Souza, Teles, 2018.

Ferreira, Veras, Brito, Gomes, Mendes, Aquino, 2014.

Soares, Lopes, 2011.

Nery, Mendonça

Gomes, Fernandes, Oliveira, 2011.

Diniz, Koller, 2012. 2019.
Pinheiro, Pereira, Freitas, Investigar os fatores associados à gravidez

Verificar causas e consequências da gravidez na adolescência.

Avaliar o cumprimento dos direitos constitucionais relativos à vida e à Saúde.

Atualizar dados de condições psicossociais das díades.

Conhecer os significados da maternidade para as adolescentes atendidas pela Estratégia de Saúde da Família (ESF), no município de Montes Claros-MG/Brasil.

Descrever as causas predisponentes à gestação entre adolescentes e seu conhecimento sobre os métodos de prevenção.

Conhecer e compreender as vivências de gestação e maternidade na adolescência em assentamentos rurais.

Identificar os fatores de reincidência da gravidez na adolescência e analisar os fatores sócio- econômicos, culturais, obstétricos e os motivos apontados pela adolescente para a reincidência de gravidez até dois anos após o término de uma gestação.

Investigar as características associadas à gravidez durante a adolescência em uma população de adolescentes brasileiros de baixa renda na adolescência.
Estudo Descritivo com

abordagem quantitativa

Estudo exploratório de natureza quantitativa,

Estudo longitudinal

Pesquisa de campo qualitativa, com abordagem descritiva.

Pesquisa exploratória, descritiva e quantitativa.

Abordagem qualitativa, sustentada em método biográfico.

Estudo interrelacional retrospectivo.

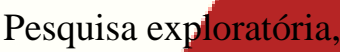
descritiva e quantitativa 


\section{Silva,}

Coutinho,

Katz, Souza, 2013

Martinez, Roza,

Guimarães, Bava

Achcar, Dal-Fabbro, 2011.

Silva, surita, 2012.

Albuquerque, Pitangui, Rodrigues, Araújo, 2017.

Santos, Silva, Queiroz, Jorge, 2018.
Identificar os fatores associados à repetição da gravidez na adolescência.

Estudar a associação entre os percentuais de gravidez na adolescência e características socioeconômicas e de vulnerabilidade social dos municípios do Estado de São Paulo, Brasil,

Investigar o índice de casos de gravidez em adolescentes.

Determinar a prevalência e fatores associados a gravidez de repetição rápida

(GRR) em gestantes adolescentes.

Compreender a trajetória de adolescentes acerca da primeira gravidez, contemplando realidades e perspectivas.
Estudo do tipo casocontrole

Estudo ecológico,

Estudo bibliográfico.

Estudo transversal

Estudo qualitativo, fundamentado no referencial teórico

Fonte: Elaborado pelos autores (2020).

Nos estudos destacados no Quadro 1 é possível observar que referente ao número de autores por estudo publicado, foi observado que, dos 14 artigos analisados, 3 apresentam dois autores, 3 apresentam três autores e o restante, apresentam quatro ou mais autores.

Esta observação evidencia a tendência que vem sendo percebida a algum tempo, que é o desenvolvimento de estudos em grupo, com a participação média de três profissionais, sendo esses de diversas formações. Nos estudos analisados, houve o predomínio de profissionais da enfermagem e medicina.

Quanto à distribuição dos artigos selecionados por ano de publicação, entre 2010 e 2019, os 14 artigos analisados foram publicados em anos diferentes, na seguinte ordem de publicação 2010 - (1), 2011 - (3), 2012 - (2), 2013 - (1), 2014- (2), 2017 - (1), 2018 - (2) e por fim 2019 $-(1)$.

A leitura dos temas abordados pelas publicações analisadas, permitiu evidenciar aspectos gerais, relacionados como fatores predisponentes para gravidez na adolescência, sendo eles os fatores econômicos, sociais, emocionais e psicológicos.

Estes achados indicam que o número de filhos, o exercício de atividade remunerada e o uso de métodos contraceptivos são fatores protetores importantes para evitar a adolescência. Em contraste, o não planejamento da gravidez aumentou demasiadamente a probabilidade de gestação em mulheres menores que 19 anos (PINHEIRO, PEREIRA, FREITAS, 2019; SANTOS, et al, 2018). 
Albuquerque et al. (2017), em seu estudo destacam que a maioria das adolescentes estudadas tinham 16 anos e que, esse fato foi recorrente nas adolescentes que tiveram mais de uma gestão durante sua adolescência em menos de dois anos a partir de sua última gravidez.

A média de idade relatada por Albuquerque et al. (2017), é recorrente em outros estudos como o de Ferreira et al. (2014), em entrevista com mães adolescente a idade variou de 14 a 19 anos, tendo uma média de 16,8 anos. Nery et al. (2011), relatam que (435) 88,5\% de suas entrevistadas tinha idade média de 15 a 19 anos quando engravidaram e (120) 25,9\% delas tiveram gravidez reincidente em pelo menos dois anos.

Silva et al. (2013), apontam que a Idade da coitarca inferior a 15 anos, ter engravidado pela primeira vez antes dos 16 anos e não amamentar após a última gestação foram as características reprodutivas e de assistência à saúde que se associaram a uma maior chance de recorrência de gravidez em adolescência.

Martinez et al. (2011), verificaram que a ocorrência de gravidez dos 15 aos 19 anos de idade é maior na zona rural do que nas áreas metropolitanas, onde de forma geral, há mais acesso à educação e à informação.

Silva et al. (2013) observaram que entre as adolescentes com gestação recorrente a chance de a primeira gestação ter acontecido antes dos 16 anos foi aproximadamente três vezes maior. A idade na primeira gestação já caracteriza que não houve cuidados preventivos e quanto mais cedo e mais imatura a adolescente, menor a probabilidade de mudança desse comportamento.

A situação econômica de familiares da adolescente, traduzida pela renda familiar, foi descrita por outros autores como um fator importante para ocorrência de gestação durante a adolescência (SILVA et al., 2013). Albuquerque et al. (2017), apontam a renda familiar de todas as adolescentes estudadas era de até dois salários mínimos. Alguns autores atentam para o fato de que a gravidez seja perpetuadora da baixa condição socioeconômica.

Carmo et al. (2014), relatam que 92,4\% das jovens entrevistadas em seu estudo, não estavam trabalhando no momento da pesquisa, complementa ainda afirmando que a po idade, em conjunto com a baixa escolaridade das adolescentes, reduz a probabilidade de inserção no mercado de trabalho, que está cada vez mais exigente, e raramente podem ser encontradas em mães adolescentes, das que conseguem empregos são mal remuneradas, o que as deixa dependentes financeiramente de familiares e/ou parceiro.

A baixa escolaridade, pode ser um dos fatores colaboradores ocorrência da gravidez, após se tornarem mãe, não conseguem retornar à escola pa comprometendo dessa forma sua perspectiva de futuro, o que acaba perpetuando o ciclo de 
pobreza e com gravidezes recorrentes elas permanecem definitivamente fora da escola (NERY, et al., 2011).

Martinez et al. (2011), em um estudo sobre indicadores sociais em uma população de gestantes, estimaram que apenas 6,8\% das adolescentes eram estudantes. Entretanto, se por um lado a gravidez na adolescência pode ocasionar a evasão escolar, muitas adolescentes podem ter deixado a escola antes de engravidar, sendo por sua vez esta evasão um provável fator de risco para a gravidez precoce.

Martinez et al. (2011), ressaltam que filhos adolescentes de mulheres com um ano de escolaridade têm probabilidade 23 vezes maior de chegar analfabeto à adolescência, em comparação com filhos de mulheres com 11 anos ou mais de escolaridade.

A baixa escolaridade e a baixa renda são consideradas importantes causas da gravidez na adolescência, apontando uma alta proporção de mulheres grávidas que possuem baixa escolaridade ( $<8$ anos de estudo) e baixa renda ( $<1 \mathrm{SM})$. Entretanto, é evidenciado que essa variável é uma constante no que diz respeito a mulheres adolescentes e adultas. (PINHEIRO, PEREIRA, FREITAS, 2019).

A pobreza, associada ao baixo nível de escolaridade, são dois fatores que estão intrinsecamente relacionados, qualquer que seja o estado civil da adolescente, a maioria é pobre ou sem fontes adequadas, algumas porque são solteiras e/ou estão na escola, outras porque estão casadas, não trabalham ou têm salários extremamente baixos, as adolescentes são induzidas ao casamento muito cedo e encorajadas a ficarem grávidas o quanto antes, uma vez que a maioria é desprovida de perspectivas de futuro e não conhece outra vida, que não tenha como foco a maternidade (SILVA; SURITA, 2012).

\section{CONCLUSÕES}

Tendo como base as informações acima citadas, é possível concluir que as principai causas da gravidez na adolescência podem ser organizadas em três grandes aspectos principais que se correlacionam, são eles social, econômico e aspecto emocional e psicológico. Foi observado que a maioria das adolescentes em situações de vulnerabilidade socioeconômica são as mais afetadas com esse problema de saúde pública.

As jovens que estão inseridas nesse contexto apresentam um nível baixo de escolaridade associado a uma renda familiar de até dois salários mínimos. São meninas que não tem uma perspectiva promissora para o futuro. Ainda relacionado a estes fatores é importante ressaltar que a maioria das adolescentes que engravidaram não tinha um bom relacionamento familiar, 
fazendo com que criassem meios para sair de casa o mais rápido possível, mesmo que o único jeito fosse realizando uma atitude totalmente irresponsável.

Em todos os artigos analisados foi possível perceber a ausência de políticas públicas que ampare essas adolescentes ou até mesmo uma medida para evitar que a gravidez aconteça. Não existe um preparo da equipe de saúde para lidar com essa situação.

Se tratando de um problema de saúde pública, são necessárias novas abordagens capazes de direcionar o atendimento para essas adolescentes de forma multidisciplinar, permitindo à essas jovens a visualização de novos horizontes, não sendo a gravidez o único "recurso" para mudança de vida.

\section{REFERÊNCIAS}

ALBUQUERQUE, Ana Paula dos Santos. et al.. Prevalência da gravidez de repetição rápida e fatores associados em adolescentes de Caruaru, Pernambuco. Rev. Bras. Saude Mater. Infant. Recife, v. 17, n. 2, p. 347-354, Jun 2017. Disponível em <http://www.scielo.br/scielo.php?script=sci_arttext\&pid=S1519$38292017000200347 \& \operatorname{lng}=\mathrm{en} \& n r m=i s o>$. Acesso em 08 de maio de 2020.

BERNI, Vanessa Limana; ROSO, Adriane. A adolescência na perspectiva da psicologia social crítica. Psicol. Soc. , Belo Horizonte, v. 26, n. 1, pág. 126-136, abril de 2014. Disponível em <http://www.scielo.br/scielo.php?script=sci_arttext\&pid=S0102-

$71822014000100014 \& \operatorname{lng}=\mathrm{en} \& n r m=$ iso $>$. Acesso em 03 de julho de 2020.

BRASIL. Ministério da Saúde. Indicadores e dados básicos: sistema de informações sobre nascidos vivos. (Sinasc) 2. ed. - Brasília: Organização Pan-Americana da Saúde, 2008. 349 p. Disponível: < http://tabnet.datasus.gov.br/tabdata/livroidb/2ed/indicadores.pdf>. Acesso em 05 junho de 2020.

Ministério da Saúde do Brasil. Brasil acelera redução de gravidez na adolescência. Capturado 19 set. 2010. Disponível em: <http://portal.saude.gov.br/portal/aplicacoes/noticias/default.cfm?


2020.

Lei 8.069, de 13 de julho de 1990. Estatuto da Criança e do Adolescente. Brasília:

Ministério da Justiça, 1990. Disponível:

http://www.planalto.gov.br/ccivil_03/leis/18069.htm>. Acesso em 05 junho de 2020.

BRÊTAS, J, R, S. Mudanças: A corporalidade na adolescência. 264 f. (Tese de doutorado em enfermagem) - Universidade Federal de São Paulo, 2003. Disponível https://core.ac.uk/download/pdf/37721755.pdf>. Acesso em 29 de julho de 2020.

BRITO, Isabel. Ansiedade e depressão na adolescência. Rev Port Clin Geral, Lisboa, v. 27, n. 2, p. 208-214, mar. 2011. Disponível <http://www.scielo.mec.pt/scielo.php?script=sci_arttext\&pid=S0870$71032011000200010 \& \operatorname{lng}=$ pt\&nrm=iso>. Acesso em 06 de junho 2020 
CARMO, Suelen Souza do. et al. Análise quantitativa sobre gravidez na adolescência em um município mineiro. Cogitare enferm. Curitiba, v. 19, n. 4, p. 801-807, dez. 2014

Disponível em <http://www.revenf.bvs.br/scielo.php?script=sci_arttext\&pid=S1414$85362014000400021 \& \operatorname{lng}=$ pt\&nrm=iso>. Acesso em 07 de julho de 2020.

COSTA, Vanuzia; FERNANDES, Sheyla Christine Santos. O que pensam os adolescentes sobre o amor e o sexo? Um estudo na perspectiva das representações sociais. Psicol. Soc., Belo Horizonte, v. 24, n. 2, pág. 391-401, agosto de 2012. Disponível em <http://www.scielo.br/scielo.php?script=sci_arttext\&pid=S0102-

$71822012000200017 \& \operatorname{lng}=e n \& n r m=i$ so $>$. Acesso em 06 de junho de 2020.

CHAUI, M. Repressão Sexual: essa nova (des) conhecida. São Paulo: Brasiliense, 1987.

DIAS, Ana Cristina Garcia; TEIXEIRA, Marco Antônio Pereira. Gravidez na adolescência: um olhar sobre um fenômeno complexo. Paidéia (Ribeirão Preto), Ribeirão Preto, v. 20, n. 45, pág. 123-131, abril de 2010. Disponível em <http://www.scielo.br/scielo.php?script=sci_arttext\&pid=S0103863X2010000100015\&lng=en\&nrm=iso>. Acesso em 01 de setembro de 2020.

DINIZ, Eva; KOLLER, Silvia Helena. Fatores associados à gravidez em adolescentes brasileiros de baixa renda. Paidéia (Ribeirão Preto), Ribeirão Preto, v. 22, n. 53, pág. 305314, dezembro de 2012. Disponível em <http://www.scielo.br/scielo.php?script=sci_arttext\&pid=S0103-

863X2012000300002\&lng=en\&nrm=iso>. Acesso em 08 de setembro de 2020.

EISENSTEIN, E. Adolescência: definições, conceitos e critérios. Revista Adolescência e Saúde. Vila Isabel, v.2, n.2, pag. 6-7, Abr/Jun - 2005. Disponível em: $<$ https://cdn.publisher.gn1.link/adolescenciaesaude.com/pdf/v2n2a02.pdf >. Acesso em 03 de junho de 2020.

ERIKSON, E. Identidade, juventude e crise. $2^{\text {a }}$ ed. Rio de Janeiro: Zahar, 1976.

FIEDLER, Milla Wildemberg; ARAÚJO, Alisson; SOUZA, Márcia Christina Caetano de. A prevenção da gravidez na adolescência na visão de adolescentes. Texto \& Contexto Enfermagem, vol. 24, n. 1, jan, 2015, pag. 30-37. Disponível: < https://www.redalyc.org/articulo.oa?id=71438421004>. Acesso em 01 junho de 2020.

FILIPINI, C,B. et al. Transformações físicas e psíquicas: um olhar do adolescente. Adolesc Saude, Rio de Janeiro v. 10, n.1 2013. Pag. 22-29. . Disponível: http://www.adolescenciaesaude.com/detalhe_artigo.asp?id=351>. Acesso em 01 junho de 2020.

LLOYD. Cynthia, B; Conselho Nacional de Pesquisa e Instituto de Medicina. Growing Up Global: The Changing Transitions to Adulthood in Developing countries. Washington, DC: The National Academies Press. 2005. Disponível $<$ https://www.nap.edu/catalog/11174/growing-up-global-the-changing-transitions-toadulthood-in-developing> acesso em 08 de junho de 2020.

MARTINEZ, Edson Zangiacomi. et al. Gravidez na adolescênci socioeconômicas dos municípios do Estado de São Paulo, Brasil: anátise cspa Pública, Rio de Janeiro, v. 27, n. 5, p. 855-867, maio 2011. 
$<$ http://www.scielo.br/scielo.php?script=sci_arttext\&pid=S0102-

311X2011000500004\&lng=pt\&nrm=iso>. Acesso em 08 setembro de 2020.

OLIVEIRA, Carla Renata Faria de. A adolescência e a gravidez precoce nas famílias de baixa renda. 2018. 63 f. Monografia (especialização em saúde da família) - Universidade Candido Mendes, Rio das Ostras, 2018. Disponível em: < https://www.avm.edu.br/docpdf/monografias_publicadas/posdistancia/53845.pdf >. Acesso em 29 de julho de 2020.

ONYEONORO, U, U. et al. Sources of sex information and its effects on sexual practices among in-school female adolescents in Osisioma Ngwa LGA, South East Nigeria. J Pediatr Adolesc Gynecol. V, 24, ed. 5, Pag. 294-299, outubro de 2011. Disponível em < https://www.jpagonline.org/article/S1083-3188(11)00230-0/fulltext>. Acesso em 06 de junho de 2020.

PINHEIRO, Yago Tavares; PEREIRA, Natália Herculano; FREITAS, Giane Dantas de Macêdo. Fatores associados à gravidez em adolescentes de um município do nordeste do Brasil. Cad. saúde colet., Rio de Janeiro, v. 27, n. 4, p. 363-367, Dec. 2019. Disponível em: $<$ http://www.scielo.br/scielo.php?script=sci_arttext\&pid=S1414-

462X2019000400363\&lng=en\&nrm=iso>. Acesso em 07 junho. 2020.

ROSSETTO, Micheli Scolari; SCHERMANN, Lígia Braun; BERIA, Jorge Umberto. Maternidade na adolescência: indicadores emocionais negativos e fatores associados em mães de 14 a 16 anos em Porto Alegre, RS, Brasil. Ciênc. Saúde coletiva, Rio de Janeiro, v. 19, n. 10, p. 4235-4246, out. 2014. Disponível em: $<$ http://www.scielo.br/scielo.php?script=sci_arttext\&pid=S1413-

81232014001004235\&lng=en\&nrm=iso>. Acesso em 07 junho. 2020.

SANTOS, Rita de Cássia Andrade Neiva, et al. Realidades e perspectivas de mães adolescentes acerca da primeira gravidez. Rev. Bras. Enferm., Brasília , v. 71, n. 1, p. 6572, Feb. 2018. Available from

$<$ http://www.scielo.br/scielo.php?script=sci_arttext\&pid=S0034-

$71672018000100065 \& \operatorname{lng}=\mathrm{en} \& \mathrm{nrm}=\mathrm{iso}>$. Acesso em 08 de setembro de 2020.

SENNA, Sylvia Regina Carmo Magalhães; DESSEN, Maria Auxiliadora. Reflexões sobre a saúde do adolescente brasileiro. Psic., Saúde \& Doenças, Lisboa , v. 16, n. 2, p. 217 229, set. $2015 \quad$ Disponível <http://www.scielo.mec.pt/scielo.php?script=sci_arttext\&pid=S1645$00862015000200008 \& \operatorname{lng}=$ pt\&nrm=iso $>$. Aceso em 03 de agosto 2020.

SILVA, Aniel de Sarom Negrão et al. Início da vida sexual em adolescentes escolares: un estudo transversal sobre comportamento sexual de risco em Abaetetuba, Estado do Brasil. Rev Pan-Amaz Saude, Ananindeua, v. 6, n. 3, p. 27-34, set. 2015. . Disponível em $<$ http://scielo.iec.gov.br/scielo.php?script=sci_arttext\&pid=S2176$62232015000300004 \& \operatorname{lng}=$ pt\&nrm=iso $>$. Acesso em 07 junho de 2020.

SILVA, Andréa de Albuquerque Arruda et al.Fatores associados à recorrência da adolescência em uma maternidade escola: estudo caso-controle. Cad. Saúde Púl Janeiro, v. 29, n. 3, p. 496-506, mar. 2013. <http://www.scielo.br/scielo.php?script=sci_arttext\&pid=S0102$311 X 2013000300008 \& \operatorname{lng}=$ en\&nrm=iso>. Acesso em 08 de setembro de 2020 
SILVA, João Luiz Pinto e; SURITA, Fernanda Garanhani CASTRO. Gravidez na adolescência: situação atual. Rev. Bras. Ginecol. Obstet., Rio de Janeiro, v. 34, n. 8, pág. 347350, agosto de 2012. Disponível em $<$ http://www.scielo.br/scielo.php?script=sci_arttext\&pid=S0100-

$72032012000800001 \& \operatorname{lng}=$ en\&nrm=iso>. Acesso em 08 de setembro de 2020.

SOARES, Joannie dos Santos Fachinelli; LOPES, Marta Julia Marques. Biografias de gravidez e maternidade na adolescência em assentamentos rurais no Rio Grande do Sul. Rev. esc. enferm. USP, São Paulo, v. 45, n. 4, p. 802-810, ago. 2011. Disponível em: $<$ http://www.scielo.br/scielo.php?script=sci_arttext\&pid=S0080-

$62342011000400002 \& \operatorname{lng}=\mathrm{en} \& n r m=$ iso $>$. Acesso em 07 de maio de 2020.

SOUZA, Marcela Tavares de; SILVA, Michelly Dias da; CARVALHO, Rachel de. Revisão integrativa: o que é e como fazer. Einstein (São Paulo), São Paulo, v. 8, n. 1, p. 102-106, mar. 2010 . Disponível em <http://www.scielo.br/scielo.php?script=sci_arttext\&pid=S1679$45082010000100102 \& \operatorname{lng}=\mathrm{en} \& n r m=\mathrm{iso}>$. Acesso em 03 de junho 2020.

VALLE, Luiza Elena L. Ribeiro do; MATTOS, Maria José Viana Marinho de. Adolescência: as contradições da idade. Rev. psicopedag., São Paulo , v. 28, n. 87, p. 321-323, 2011

Disponível em: <http://pepsic.bvsalud.org/scielo.php?script=sci_arttext\&pid=S0103$84862011000300012 \& \operatorname{lng}=$ pt\&nrm=iso>. Acesso em 21 agosto de 2020.

VANZIN, Rafaela. et al. Vida sexual de adolescentes escolares da rede pública de Porto VelhoRO. Aletheia, Canoas, n. 41, p. 109-120, ago. 2013 . Disponível em <http://pepsic.bvsalud.org/scielo.php?script=sci_arttext\&pid=S1413-

03942013000200009\&lng=pt\&nrm=iso>. Acesso em 07 junho de 2020.

VIOLA, Daniela Teixeira Dutra; VORCARO, Ângela Maria Resende. A adolescência em perspectiva: Um exame da variabilidade da passagem à idade adulta entre diferentes sociedades. Psic.: Teor. e Pesq., Brasília , v. 34, e3448, 2018 . Disponível: $<$ http://www.scielo.br/scielo.php?script=sci_arttext\&pid=S0102-

$37722018000100507 \& \operatorname{lng}=$ en\&nrm=iso $>$. Acesso em 01 junho de 2020.

WHO. World Health Organization. Child and Adolescent Health Development. Geneva: WHO; 2001. Disponível em: <http:// www.who.int/child-adolescenthealth/OVERVIEW/AHD/adhover.htm>. Acesso em: 06 de maio de 2020. 